\title{
Spiking Neural Network Decoder for Brain-Machine Interfaces
}

\author{
Julie Dethier, Student Member, IEEE, Vikash Gilja, Member, IEEE, Paul Nuyujukian, Student Member, IEEE, \\ Shauki A. Elassaad, Member, IEEE, Krishna V. Shenoy, Senior Member, IEEE \\ and Kwabena Boahen, Senior Member, IEEE
}

\begin{abstract}
We used a spiking neural network (SNN) to decode neural data recorded from a 96-electrode array in premotor/motor cortex while a rhesus monkey performed a point-to-point reaching arm movement task. We mapped a Kalman-filter neural prosthetic decode algorithm developed to predict the arm's velocity on to the SNN using the Neural Engineering Framework and simulated it using Nengo, a freely available software package. A 20,000-neuron network matched the standard decoder's prediction to within $0.03 \%$ (normalized by maximum arm velocity). A 1,600-neuron version of this network was within $0.27 \%$, and run in real-time on a $3 \mathrm{GHz}$ PC. These results demonstrate that a SNN can implement a statistical signal processing algorithm widely used as the decoder in high-performance neural prostheses (Kalman filter), and achieve similar results with just a few thousand neurons. Hardware SNN implementations-neuromorphic chips-may offer power savings, essential for realizing fully-implantable cortically controlled prostheses.
\end{abstract}

\section{Cortically-Controlled Motor Prostheses}

Neural prostheses aim to restore functions lost to neurological disease and injury. Motor prostheses aim to help disabled patients by translating neural signals from the brain into control signals for prosthetic limbs or computer cursors. We recently reported a closed-loop cortically-controlled motor prosthesis capable of producing quick, accurate, and robust computer cursor movements by decoding action potentials from a 96-electrode array in rhesus macaque premotor/motor cortex [1]-[4]. This design and previous high-performance designs as well (e.g., [5]) employ versions of the Kalman filter, ubiquitous in statistical signal processing.

While these recent advances are encouraging, true clinical viability awaits fully-implanted systems which, in turn, impose severe power dissipation constraints. For example, to avoid heating the brain by more than $1^{\circ} \mathrm{C}$, which is believed to be important for long term cell health, a $6 \times 6 \mathrm{~mm}^{2}$

This work was supported in part by the Belgian American Education Foundation (J. Dethier), NSF and NDSEG Graduate Research Fellowships (V. Gilja), Stanford NIH Medical Scientist Training Program (MSTP) and Soros Fellowship (P. Nuyujukian), DARPA Revolutionizing Prosthetics program (N66001-06-C-8005, K. V. Shenoy), and two NIH Director's Pioneer Awards (DP1-OD006409, K. V. Shenoy; DPI-OD000965, K. Boahen).

J. Dethier, S. A. Elassaad and K. Boahen are with the Department of Bioengineering, Stanford University, Stanford, CA 94305, USA (email: [jdethier, shauki, boahen]@stanford.edu).

V. Gilja is with the Department of Computer Science and Stanford Institute for Neuro-Innovation and Translational Neuroscience, Stanford University, Stanford, CA 94305, USA (email: gilja@stanford.edu).

P. Nuyujukian is with the Department of Bioengineering and MSTP, Stanford University, Stanford, CA 94305, USA (email: paul@npl.stanford.edu).

K. V. Shenoy is with the Departments of Electrical Engineering and Bioengineering, and Neurosciences Program, Stanford University, Stanford, CA 94305, USA (email: shenoy@ stanford.edu). implant must dissipate less than $10 \mathrm{~mW}$ [6]. Running the 96electrode to 2 degree-of-freedom Kalman-filter on a $3.06 \mathrm{GHz}$ Core Duo Intel processor took $0.985 \mu$ s/update, or 6,030 flops/update, which, at $66.3 \mathrm{Mflops} /$ watt, consumes $1.82 \mathrm{~mW}$ for 20 updates/sec. This lack of low-power circuits for neural decoding is a major obstacle to the successful translation of this new class of motor prostheses.

We focus here on a new approach to implementing the Kalman filter that is capable of meeting these power constraints: the neuromorphic approach. The neuromorphic approach combines digital's and analog's best featuresprogrammability and efficiency-offering potentially greater robustness than either [7], [8]. At 50nW per silicon neuron [9], a neuromorphic chip with 1,600 spiking neurons would consume $80 \mu \mathrm{W}$. To exploit this energy-efficient approach to build a fully implantable and programmable decoder chip, the first step is to explore the feasibility of implementing existing decoder algorithms with spiking neural networks (SNN) in software. We did this for the Kalman-filter based decoder [1]-[4] using Nengo, a freely available simulator [10].

\section{KALMAN-FILTER DeCODER}

The concept behind the Kalman filter is to track the state of a dynamical system throughout time using a model of its dynamics as well as noisy measurements. The model gives an estimate of the system's state at the next time step. This estimate is then corrected using the measurements at this time step. The relative weights for these two pieces of information are given by the Kalman gain, K [11], [12].

For neural applications, the cursor's kinematics define the system's state vector, $\mathbf{x}_{t}=\left[\right.$ vel $_{t}^{\mathrm{x}}$, vel $\left._{t}^{\mathrm{y}}, 1\right]$; the constant 1 allows for a fixed offset compensation. The neural spike rate (spike counts in each time step) of 96 channels of action-potential threshold crossings defines the measurements vector, $\mathbf{y}_{t}$. And the system's dynamics are modeled by:

$$
\begin{aligned}
\mathbf{x}_{t} & =\mathbf{A} \mathbf{x}_{t-1}+\mathbf{w}_{t}, \\
\mathbf{y}_{t} & =\mathbf{C} \mathbf{x}_{t}+\mathbf{q}_{t},
\end{aligned}
$$

where $\mathbf{A}$ is the state matrix, $\mathbf{C}$ is the observation matrix, and $\mathbf{w}_{t}$ and $\mathbf{q}_{t}$ are additive, Gaussian noise sources with $\mathbf{w}_{t} \sim$ $N(0, \mathbf{W})$ and $\mathbf{q}_{t} \sim N(0, \mathbf{Q})$. The model parameters (A, $\mathbf{C}, \mathbf{W}$ and $\mathbf{Q}$ ) are fit with training data.

Assuming the system is stationary, we estimate the current system state by combining the estimate at the previous time step with the noisy measurements using the Kalman gain $\mathbf{K}=\left(\mathbf{I}+\mathbf{W C Q}^{-1} \mathbf{C}\right)^{-1} \mathbf{W} \mathbf{C} \mathbf{Q}^{-1}$. This yields:

$$
\hat{\mathbf{x}}_{t}=(\mathbf{I}-\mathbf{K C}) \mathbf{A} \hat{\mathbf{x}}_{t-1}+\mathbf{K} \mathbf{y}_{t} \text {. }
$$




\section{NeUral ENGINEERING FrameWORK}

Neural engineers have developed a formal methodology for mapping control-theory algorithms onto a computational fabric consisting of a highly heterogeneous population of spiking neurons simply by programming the strengths of their connections [10]. These artificial neurons are characterized by a nonlinear multi-dimensional-vector-to-spikerate function $-a_{i}(\mathbf{x}(t))$ for the $i^{\text {th }}$ neuron-with parameters (preferred direction, gain, and threshold) drawn randomly from a wide distribution (standard deviation $\approx$ mean).

The neural engineering approach to configuring SNNs to perform arbitrary computations involves representation, transformation, and dynamics [10], [13]-[15]:

- Representation is defined by nonlinear encoding of $\mathbf{x}(t)$ as a spike rate, $a_{i}(\mathbf{x}(t))$, combined with weighted linear decoding of $a_{i}(\mathbf{x}(t))$ to recover an estimate of $\mathbf{x}(t), \hat{\mathbf{x}}(t)=\sum_{i} a_{i}(\mathbf{x}(t)) \phi_{i}^{\mathbf{x}}$. The decoding weights, $\phi_{i}^{\mathbf{x}}$, are obtained by minimizing the mean squared error.

- Transformation is performed by using alternate decoding weights in the decoding operation to map transformations of $\mathbf{x}(t)$ directly into transformations of $a_{i}(\mathbf{x}(t))$. For example, $\mathbf{y}(t)=\mathbf{A x}(t)$ is represented by the spike rates $b_{j}(\mathbf{A} \hat{\mathbf{x}}(t))$, where unit $j$ 's input is computed directly from unit $i$ 's output using $\mathbf{A} \hat{\mathbf{x}}(t)=\sum_{i} a_{i}(\mathbf{x}(t)) \mathbf{A} \phi_{i}^{\mathbf{x}}$, an alternative linear weighting.

- Dynamics are realized by using the synapses' spike response, $h(t)$, (aka, impulse response) to capture the system's dynamics. For example, for $h(t)=\tau^{-1} e^{-t / \tau}$, $\dot{\mathbf{x}}=\mathbf{A x}(t)$ is realized by replacing $\mathbf{A}$ with $\mathbf{A}^{\prime}=\tau \mathbf{A}+$ I. This so-called neurally plausible matrix yields an equivalent dynamical system: $\mathbf{x}(t)=h(t) * \mathbf{A}^{\prime} \mathbf{x}(t)$, where convolution replaces integration.

The nonlinear encoding process-from a multidimensional stimulus, $\mathbf{x}(t)$, to a one-dimensional soma current, $J_{i}$, to a firing rate, $a_{i}(\mathbf{x}(t))$-is specified as:

$$
a_{i}(\mathbf{x}(t))=G\left(J_{i}(\mathbf{x}(t))\right)
$$

Here $G()$ is the neurons' nonlinear current-to-spike-rate function, which is given by

$$
G\left(J_{i}(\mathbf{x})\right)=\left\{\tau^{\mathrm{ref}}-\tau^{\mathrm{RC}} \ln \left(1-J_{\mathrm{th}} / J_{i}(\mathbf{x})\right)\right\}^{-1},
$$

for the leaky integrate-and-fire model (LIF). This model's subthreshold behavior is described by an RC circuit with time constant $\tau^{\mathrm{RC}}$. When the voltage reaches the threshold, $V_{\mathrm{th}}$, the neuron emits a spike $\delta\left(t-t_{n}\right)$. After this spike, the neuron is reset and rests for $\tau^{\text {ref }}$ seconds (absolute refractory period) before it resumes integrating. $J_{\mathrm{th}}=V_{\mathrm{th}} / \mathrm{R}$ is the minimum input current that produces spiking. Ignoring the soma's RC time-constant when specifying the SNN's dynamics is reasonable because the neurons cross threshold at a rate that is proportional to their input current, which thus sets the spike rate instantaneously, without any filtering [10].

The conversion from a multi-dimensional stimulus, $\mathbf{x}(t)$, to a one-dimensional soma current, $J_{i}$, is performed by
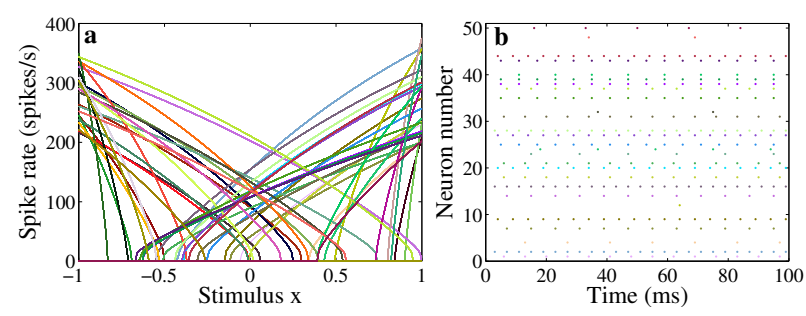

Fig. 1. a. 1D tuning curves of a population of 50 leaky integrate-and-fire neurons. The maximum firing rate and $x$-intercept are chosen from uniform distributions with range $200 \mathrm{~Hz}$ to $400 \mathrm{~Hz}$ and -1 to +1 , respectively. b. The neurons' spike responses to a stimulus $x=0.5$ (same color code).

assigning to the neuron a preferred direction, $\tilde{\phi}_{i}^{\mathbf{x}}$, in the stimulus space and taking the dot-product:

$$
J_{i}(\mathbf{x}(t))=\alpha_{i}\left\langle\tilde{\phi}_{i}^{\mathbf{x}} \cdot \mathbf{x}(t)\right\rangle+J_{i}^{\text {bias }}
$$

where $\alpha_{i}$ is a gain or conversion factor, and $J_{i}^{\text {bias }}$ is a bias current that accounts for background activity. For a 1D space, $\tilde{\phi}_{i}^{\mathbf{x}}$ is either 1 or -1 (drawn randomly). For a 2D space, $\tilde{\phi}_{i}^{\mathbf{x}}$ is uniformly distributed on the unit circle. The resulting tuning curves and spike responses are illustrated in Fig. 1 for $1 \mathrm{D}$. The information lost by decoding this nonlinear representation using simple linear weighting is not severe, and can be alleviated by increasing the population size [10].

\section{Kalman Filter with Spiking NeUrons}

To implement the Kalman filter with a SNN by applying the Neural Engineering Framework (NEF), we first convert (3) from discrete time (DT) to continuous time (CT), then we replace the CT matrices with neurally plausible ones, and use them to specify the SNN's weights (Fig. 2). This yields:

$$
\mathbf{x}(t)=h(t) *\left(\mathbf{A}^{\prime} \mathbf{x}(t)+\mathbf{B}^{\prime} \mathbf{y}(t)\right),
$$

where

$$
\begin{aligned}
& \mathbf{A}^{\prime}=\tau \mathbf{M}_{x}^{\mathrm{CT}}+\mathbf{I}=\frac{\tau}{\Delta t}\left(\mathbf{M}_{x}^{\mathrm{DT}}-\mathbf{I}\right)+\mathbf{I}, \\
& \mathbf{B}^{\prime}=\tau \mathbf{M}_{y}^{\mathrm{CT}}=\frac{\tau}{\Delta t} \mathbf{M}_{y}^{\mathrm{DT}} .
\end{aligned}
$$

$\mathbf{M}_{x}^{\mathrm{DT}}=(\mathbf{I}-\mathbf{K C}) \mathbf{A}$ and $\mathbf{M}_{y}^{\mathrm{DT}}=\mathbf{K}$ are the Kalman matrices, $\Delta t$ is the discrete time step $(50 \mathrm{~ms})$, and $\tau$ is the synaptic time constant.

The $j^{\text {th }}$ neuron's input current (see (6)) is computed from the system's current state, $\mathbf{x}(t)$, which is computed from estimates of the system's previous state $\left(\hat{\mathbf{x}}(t)=\sum_{i} a_{i}(t) \phi_{i}^{\mathbf{X}}\right)$ and current input $\left(\hat{\mathbf{y}}(t)=\sum_{k} b_{k}(t) \phi_{k}^{\mathbf{y}}\right)$ using (7). This yields:

$$
\begin{aligned}
& \alpha_{j}\left\langle\tilde{\phi}_{j}^{\mathbf{x}} \cdot \mathbf{x}(t)\right\rangle+J_{j}^{\text {bias }} \\
= & \alpha_{j}\left\langle\tilde{\phi}_{j}^{\mathbf{x}} \cdot h(t) *\left(\mathbf{A}^{\prime} \hat{\mathbf{x}}(t)+\mathbf{B}^{\prime} \hat{\mathbf{y}}(t)\right)\right\rangle+J_{j}^{\text {bias }} \\
= & \alpha_{j}\left\langle\tilde{\phi}_{j}^{\mathbf{x}} \cdot h(t) *\left(\mathbf{A}^{\prime} \sum_{i} a_{i}(t) \phi_{i}^{\mathbf{x}}+\mathbf{B}^{\prime} \sum_{k} b_{k}(t) \phi_{k}^{\mathbf{y}}\right)\right\rangle+\ldots \\
= & h(t) *\left(\sum_{i} \omega_{j i} a_{i}(t)+\sum_{k} \omega_{j k} b_{k}(t)\right)+J_{j}^{\text {bias }}
\end{aligned}
$$

where $\omega_{j i}=\alpha_{j}\left\langle\tilde{\phi}_{j}^{\mathbf{x}} \mathbf{A}^{\prime} \phi_{i}^{\mathbf{x}}\right\rangle$ and $\omega_{j k}=\alpha_{j}\left\langle\tilde{\phi}_{j}^{\mathbf{x}} \mathbf{B}^{\prime} \phi_{k}^{\mathbf{y}}\right\rangle$ are the recurrent and feedforward weights, respectively. 

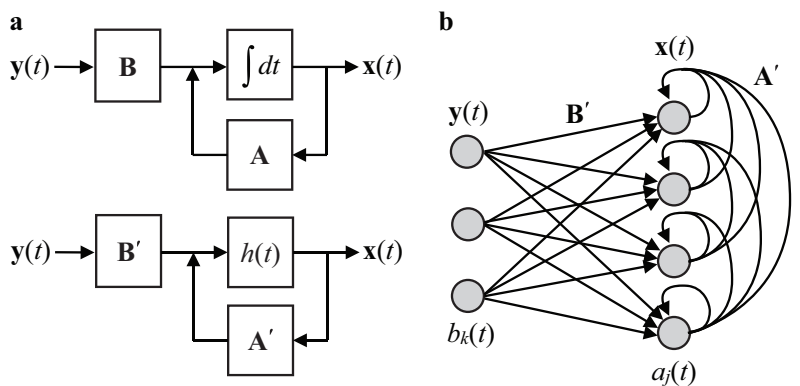

Fig. 2. Implementing a Kalman filter with spiking neurons. a. Original Kalman filter (top) and neurally plausible version (bottom). The integrator is replaced with the synapses' spike response, $h(t)$, and the matrices are replaced with $\mathbf{A}^{\prime}=\tau \mathbf{A}+\mathbf{I}$ and $\mathbf{B}^{\prime}=\tau \mathbf{B}$ to compensate. b. Spiking neural network implementation with populations $b_{k}(t)$ and $a_{j}(t)$ representing $\mathbf{y}(t)$ and $\mathbf{x}(t)$, respectively, and with feedforward and recurrent weights determined by $\mathbf{B}^{\prime}$ and $\mathbf{A}^{\prime}$, respectively.

\section{RESUlts}

An adult male rhesus macaque (monkey L) was trained to perform variants of a point-to-point arm movement task in a $3 \mathrm{D}$ experimental apparatus for juice reward [1]. ${ }^{1}$ A 96electrode silicon array (Blackrock Microsystems) was then implanted in premotor/motor cortex. Array recordings (-4.5 RMS threshold crossing applied to each electrode's signal) yielded tuned activity for the direction and speed of arm movements. As detailed in [1], a standard Kalman filter model was fit by correlating the observed hand kinematics with the simultaneously measured neural signals, while the monkey was performing the point-to-point reaching task (Fig. 3). The resulting model was used online to control an on-screen cursor in real time. This model and 500 of these trials (2010-03-08) serves as the standard against which the SNN implementation's performance is compared.

Starting with the matrices obtained by correlating the observed hand kinematics with the simultaneously measured neural signals, we built a SNN using the NEF methodology and simulated it in Nengo using the parameter values listed in Table I. We ensured that the time constants $\tau_{i}^{\mathrm{RC}}, \tau_{i}^{\mathrm{ref}}$, and $\tau_{i}^{\mathrm{PSC}}$ were smaller than the implementation's time step (50ms).

${ }^{1}$ Animal protocols were approved by the Stanford IACUC.

TABLE I

MODEL PARAMETERS

\begin{tabular}{|lll|}
\hline Symbol & Range & Description \\
\hline $\max G\left(J_{j}(\mathbf{x})\right)$ & $200-400 \mathrm{~Hz}$ & Maximum firing rate \\
$G\left(J_{j}(\mathbf{x})\right)=0$ & -1 to 1 & Normalized $\mathbf{x}$-axis intercept \\
$J_{j}^{\text {bias }}$ & Satisfies first two & Bias current \\
$\alpha_{j}$ & Satisfies first two & Gain factor \\
$\tilde{\phi}_{j}^{\mathbf{x}}$ & $\left\|\tilde{\phi}_{j}^{\mathbf{x}}\right\|=1$ & Preferred-direction vector \\
$\tau_{j}^{\text {RC }}$ & $20 \mathrm{~ms}$ & RC time constant \\
$\tau_{j}^{\text {ref }}$ & $1 \mathrm{~ms}$ & Refractory period \\
$\tau_{j}^{\text {PSC }}$ & $20 \mathrm{~ms}$ & PSC time constant \\
\hline
\end{tabular}

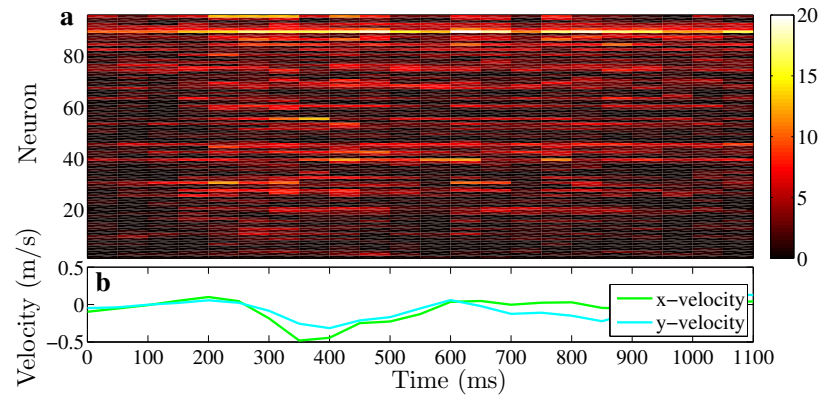

Fig. 3. Neural and kinematic measurements for one trial. a. The ninetysix cortical recordings that were fed as input to the Kalman filter and the spiking neural network (spike counts in 50ms bins). b. Arm $x$ - and $y$-velocity measurements that were correlated with the neural data to obtain the Kalman filter's matrices, which were also used to engineer the neural network.

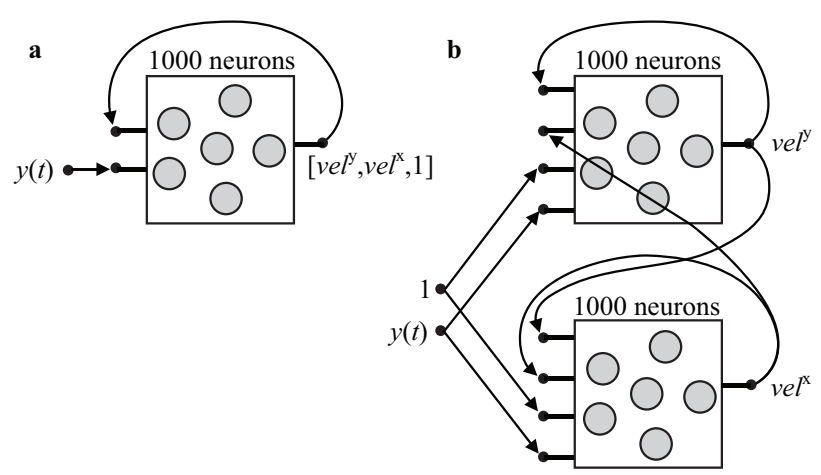

Fig. 4. Spiking neural network architectures. a. 3D integrator: A single population represents three scalar quantities $-x$ and $y$-velocity and a constant. b. 1D integrators: A separate population represents each scalar quantity $-x$ or $y$-velocity in this case.

We had the choice of two network architectures for the $a_{j}(t)$ units: a single 3D integrator or two 1D integrators (Fig. 4). The latter were more stable, as reported previously [14], and yielded better results given the available computer resources. We also had the choice of representing the 96 neural measurements with the $b_{k}(t)$ units (see Fig. 2b) or simply replacing these units' spike rates with the measurements (spike counts in $50 \mathrm{~ms}$ bins). The latter was more straight forward, avoided error in estimating the measurements, and conserved computer resources. Replacing $b_{k}(t)$ with $\mathbf{y}(t)$ 's $k^{\text {th }}$ component is equivalent to choosing $\phi_{k}^{\mathbf{y}}$ from a standard basis (i.e., a unit vector with 1 at the $k^{\text {th }}$ position and 0 everywhere else), which is what we did.

The SNN performed better as we increased the number of neurons (Fig. 5a,b). For 20,000 neurons, the $x$ and $y$ velocity decoded from its two 10,000-neuron populations matched the standard decoder's prediction to within $0.03 \%$ (RMS error normalized by maximum velocity). ${ }^{2}$ As reported in [10], the RMS error was roughly inversely proportional to the square-root of the number of neurons (Fig. 5c,d). There

${ }^{2}$ The SNN's estimates were smoothed with a filter identical to $h(t)$, but with $\tau$ set to $5 \mathrm{~ms}$ instead of $20 \mathrm{~ms}$ to avoid introducing significant delay. 

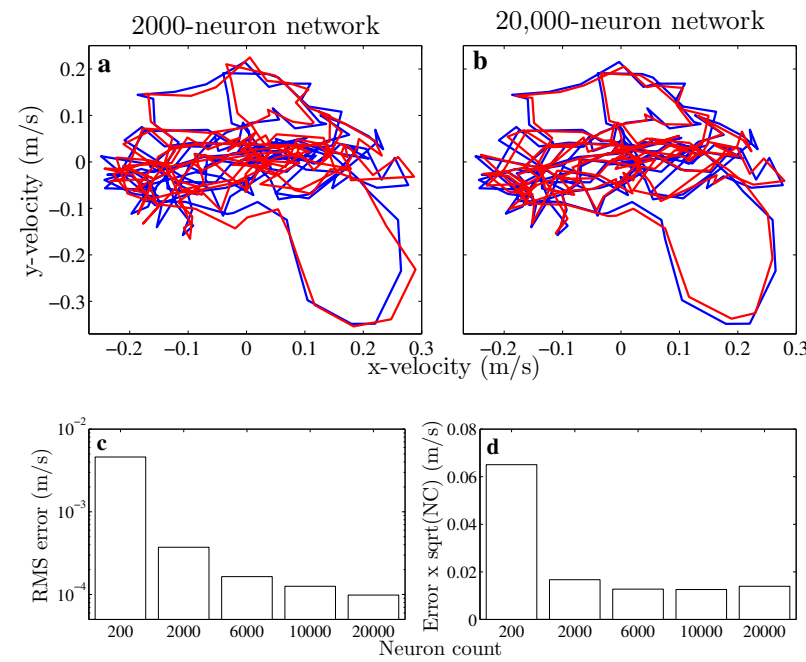

Fig. 5. Comparing the $x$ and $y$-velocity estimates decoded from 96 recorded cortical spike trains (10s of data) by the standard Kalman filter (blue) and the SNN (red). a,b. Networks with 2,000 and 20,000 spiking neurons. c. Dependence of RMS error (between SNN and Kalman filter) on network size (note log scale). d. Product of RMS error and neuron count's (NC) square root is roughly constant (for NC $>200$ ), implying that they are inversely proportional.

is a tradeoff between accuracy and computational time. For real-time operation-on a $3 \mathrm{GHz} \mathrm{PC}$ with a $1 \mathrm{~ms}$ simulation time-step - the network size is limited to 1,600 neurons. Encouragingly, this small network's error was only $0.27 \%$.

\section{CONCLUSiOnS ANd Future Work}

The Nengo simulations reported here demonstrate offline output that is virtually ident ical to that produced by a standard Kalman filter implementation. A 1,600-neuron network's output is within $0.3 \%$ of the standard implementation, and Nengo can simulate this network in real-time. Which means we can now proceed to testing our new SNN on-line. This more challenging setting will enable us to further advance the SNN implementation by incorporating recently proposed variants of the Kalman filter that have been demonstrated to further increase performance and robustness during closed-loop, real-time operation [2], [3]. As such a filter and its variants have demonstrated the highest levels of brain-machine interface performance in both human [5] and monkey users [2], these simulations provide confidence that similar levels of performance can be attained with a neuromorphic architecture. Having refined the SNN architecture, we will proceed to our final validation step: implementing the network on Neurogrid, a hardware platform with sixteen programmable neuromorphic chips that can simulate a million spiking neurons in real-time [8].

The ultimate goal of this work is to build a fully implantable and programmable decoder chip using the neuromorphic approach. Variability among the silicon neurons and the large number of synaptic connections required present challenges. A distribution of spike-rates with a CV of $15 \%$ ( $\mathrm{sigma} / \mathrm{mean})$ is typical, due to pronounced transistor mismatch in the subthreshold region where these nanopower circuits operate [9]. We have shown, however, that the NEF can effectively exploit even higher degrees of variability. Thus, the only real remaining challenge is achieving a high degree of connectivity. This one can be addressed by adopting a columnar organization, whereby nearby neurons share the same inputs, just like they do in the cortex-and in Neurogrid. This solution requires extending the NEF to a columnar architecture, a subject of ongoing research.

\section{ACKNOWLEDGMENTS}

We thank Chris Eliasmith and Terry Stewart for valuable help with Nengo.

\section{REFERENCES}

[1] V. Gilja, Towards clinically viable neural prosthetic systems, Ph.D. Thesis, Department of Computer Science, Stanford University, 2010, pp 19-22 and pp 57-73.

[2] V. Gilja, P. Nuyujukian, C.A. Chestek, J.P. Cunningham, J.M. Fan, B.M. Yu, S.I. Ryu, and K.V. Shenoy, A high-performance continuous cortically-controlled prosthesis enabled by feedback control design, 2010 Neuroscience Meeting Planner, San Diego, CA: Society for Neuroscience, 2010.

[3] P. Nuyujukian, V. Gilja, C.A. Chestek, J.P. Cunningham, J.M. Fan, B.M. Yu, S.I. Ryu, and K.V. Shenoy, Generalization and robustness of a continuous cortically-controlled prosthesis enabled by feedback control design, 2010 Neuroscience Meeting Planner, San Diego, CA: Society for Neuroscience, 2010.

[4] V. Gilja, C.A. Chestek, I. Diester, J.M. Henderson, K. Deisseroth, and K.V. Shenoy, Challenges and opportunities for next-generation intracortically based neural prostheses, IEEE TBME, 2011, in press.

[5] S.-P. Kim, J.D. Simeral, L.R. Hochberg, J.P. Donoghue, and M.J. Black, Neural control of computer cursor velocity by decoding motor cortical spiking activity in humans with tetraplegia, J Neural Engineering, vol. 5, 2008, pp 455-476.

[6] S. Kim, P. Tathireddy, R.A. Normann, and F. Solzbacher, Thermal impact of an active 3-D microelectrode array implanted in the brain, IEEE TNSRE, vol. 15, 2007, pp 493-501.

[7] K. Boahen, Neuromorphic Microchips, Scientific American, vol 292(5), 2005, pp 56-63.

[8] R. Silver, K. Boahen, S. Grillner, N. Kopell, and K.L. Olsen, Neurotech for neuroscience: Unifying concepts, organizing principles, and emerging tools, Journal of Neuroscience, vol. 27(44), 2007, pp 11807 11819.

[9] J.V. Arthur and K. Boahen, Silicon Neuron Design: The Dynamical Systems Approach, IEEE Transactions on Circuits and Systems. In press.

[10] C. Eliasmith and C.H. Anderson, Neural Engineering: Computation, representation, and dynamics in neurobiological systems., MIT Press, Cambridge, MA; 2003.

[11] R.E. Kalman, A New Approach to Linear Filtering and Prediction Problems, Transactions of the ASME-Journal of Basic Engineering, vol. 82(Series D), 1960, pp 35-45.

[12] G. Welsh and G. Bishop, An Introduction to the Kalman Filter, University of North Carolina at Chapel Hill Chapel Hill NC, vol 95(TR 95-041), 1995, pp 1-16.

[13] C. Eliasmith, A unified approach to building and controlling spiking attractor networks, Neural Computation, vol. 17, 2005, pp 1276-1314.

[14] R. Singh and C. Eliasmith, Higher-dimensional neurons explain the tuning and dynamics of working memory cells, The Journal of Neuroscience, vol. 26(14), 2006, pp 3667-3678.

[15] C. Eliasmith, How to build a brain: from function to implementation, Synthese, vol. 159(3), 2007, pp 373-388. 